\title{
Synthesis and Trypanocidal Evaluation of Some Novel 2-(Substituted benzylidene)-5, 7-dibromo-6-hydroxy-1-benzofuran-3(2H)-ones
}

\author{
K. L. Ameta ${ }^{1 *}$, Nitu S. Rathore ${ }^{1}$, Biresh Kumar ${ }^{1}$, Edith S. Malaga $\mathbf{M}^{2}$, Manuela Verastegui $P^{2}$, \\ Robert H. Gilman ${ }^{3}$, B. L. Verma ${ }^{4}$ \\ ${ }^{1}$ Department of Chemistry, FASC, Mody Institute of Technology \& Science, Lakshmangarh, India \\ ${ }^{2}$ Department of Microbiology, Faculty of Science and Philosophy, Universidad Peruana Cayetano Heredia, Lima, Peru \\ ${ }^{3}$ Department of International Health, Bloomberg School of Public Health, Johns Hopkins University, Baltimore, USA \\ ${ }^{4}$ Department of Chemistry, MLS University, Udaipur, India \\ Email: "klameta77@hotmail.com
}

Received August 7, 2012; revised September 17, 2012; accepted September 26, 2012

\begin{abstract}
Substituted 2-benzylidene-1-benzofuran-3-ones are commonly known as aurones. This class of bioactive heterocycles belongs to flavonoid family. The article intends to put forth the rational design and synthesis of a new series of aurones using 3',5'-dibromo-2', 4'-dihydroxychalcones and copper bromide in presence of DMF-water mixture (8:2, v/v) for the first time. Preliminary bioassay shows that most of compounds have good trypanocidal activity against Trypanosoma cruzi at $10 \mu \mathrm{g} / \mathrm{mL}$. Few compounds are equally potent to the standard drugs Benznidazole and Nifurtimox. The structures of the newly synthesized products 2a-n were established by elemental analysis, FTIR, ${ }^{1} \mathrm{H}$ NMR, ${ }^{13} \mathrm{C}$ NMR and mass spectroscopic studies.
\end{abstract}

Keywords: Chalcones; Aurones; Trypanosoma cruzi; Chagas Disease; Inhibition

\section{Introduction}

Substituted 2-benzylidene-1-benzofuran-3-ones are commonly known as aurones that belongs to the naturally occurring flavonoids $[1,2]$ and are structurally isomeric to flavones. They play significant role for the pigmentation of the flowers in which they are found. Antifungal, antibacterial, antiplasmodial, antileshmanial and antiviral activities of aurones have also been reported [3-6]. In view of these observations it was devised to synthesize some novel aurones using a new methodology largely on account of their trypanocidal evaluation. Chagas disease [7-10] commonly known as American trypanosomiasis is a parasitic infection caused by the $T$. cruzi. It is a serious health issue in Latin America with withering consequences in terms of human morbidity and mortality. Due to limited existing drug therapy, poor efficacy and several side effects, there is an immediate need of novel therapeutic agents for the treatment of this disease.

Chalcones, possess a wide spectrum of biological activities [11-16], offers an unprecedented opportunity to chemists to design different sized bioactive heterocycles [17-21]. The presence of $\alpha, \beta$-unsaturated carbonyl func-

${ }^{*}$ Corresponding author. tionality in chalcone makes it a versatile substrate. In continuation of our earlier venture [22-24] to design and synthesis of novel bioactive heterocycles, we herein, report a convenient one pot conversion of 3',5'-dibromo2',4'-dihydroxy substituted chalcones to 2-(substituted benzylidene)-5,7-dibromo-6-hydroxy-1-benzofuran-3(2H)ones and their trypanocidal evaluation.

\section{Results and Discussion}

\subsection{Chemistry}

Aurones have received very limited, methods of their synthesis. The most common synthetic procedures are based on their conversion of coumaran-3-ones with benzaldehydes in the presence of sodium hydroxide to afforded $Z$-aurones. $\mathrm{H}_{3} \mathrm{PO}_{4}[25]$ and acetic anhydride [26] were also used as catalysts for this conversion. Green routes, using basic alumina [27] or alumina-KF [28] have also been reported for such conversions. Oxidative cyclization of 2'-hydroxychalcones also results aurones as one of the product. Recently aurones have been synthesized from 2'-hydroxychalcones using DMSO-CuBr 2 or pyridine- $\mathrm{Hg}(\mathrm{OAc})_{2}$ [29]. To avoid the use of high boiling volatile organic solvents, more recently polyethylene 
glycol (PEG) and mercuric (II) acetate [30] were used in environmentally benign protocols for the synthesis of aurones (benzofuran-3-ones).

Presently, it is a need of more environmentally benign protocols and solvent free systems that reduces or eliminates the use of toxic or environmentally hazardous solvents. In the present study the starting 3',5'-dibromo-2', 4'-dihydroxychalcones (1a-n) were prepared by reacting 3,5-dibromo-2,4-dihydroxyacetophenone and variously substituted aromatic aldehydes in the presence of base by conventional Claisen-Schmidt condensation method by our laboratory team. 3',5'-dibromo-2',4'-dihydroxychalcones (1a-n) on refluxing (6 - $7 \mathrm{hr}$ ) with $\mathrm{CuBr}_{2}$ in presence of DMF-water (8:2, v/v) resulted 2-(substituted benzyli-dene)-5,7-dibromo-6-hydroxy-1-benzofuran-3(2H)ones (2a-n) for the first time as shown in Scheme 1, with $63 \%-73 \%$ yields. The purity of the compounds was checked by TLC and structures of the synthesized products were confirmed by their spectral and elemental analysis.

The FTIR spectrum of all the products delineated absorption bands at $1699-1713$ due to $>\mathrm{C}=\mathrm{O}$ stretching of aurones. The ${ }^{1} \mathrm{H}$ NMR spectra showed a characteristic singlet near at $\delta 6.77-6.89$ due to $1 \mathrm{H}$ of benzylidine proton while other aromatic and aliphatic protons were observed at expected regions, ${ }^{13} \mathrm{C}$ NMR spectroscopy further confirms the assigned formation of aurones. The chemical shift values in the range $\delta 186.49-192.29$ $(\mathrm{C}=\mathrm{O}), 110.15-117.41(\mathrm{CH}=\mathrm{CH})$ and were in conformity with the assigned literature values for aurones [28]. The mass spectra of the compounds showed molecular ion peak corresponding to their molecular formula. Besides this compounds showed appropriate isotopic abundances which confirmed the presence of halo groups in respective compounds. Finally the identity of synthesized aurones was established by m.p. with samples prepared by reported methods [29] and superimposable IR spectra. All newly synthesized compounds were also evaluated for their in-vitro trypanocidal evaluation.

\subsection{In Vitro Trypanocidal Evaluation}

The trypanocidal activity of compounds was evaluated by the colorimetric method based on reduction of the substrate chlorophenolred- $\beta$-d-galactopyranoside (CPRG) for $\beta$-galactosidase resulting from the expression of the gene for $T$. cruzi (Tulahuen C4) [31]. The assay was realised in 96 wells plates containing monolayer VERO cells which were infected with $5 \times 10^{4}$ trypomastigotes (Tulahuen C4). We grew the parasite using VERO cells that are infected with $T$. cruzi trypomastigotes. The parasite is in the trypomastigote stage before it infects the cells. Once it infects the Vero cells, it enters an amastigotes stage and begins to reproduce as amastigotes. When it is released from the cells, it returns to the original trypomastigote stage to infect new cells. All the ac- tive compounds showed anti-trypanocidal activity, passed through a second test for determining the inhibitory concentration of $50 \%$ growth of the parasites $\left(\mathrm{IC}_{50}\right)$. These compounds were evaluated at $10,2,0.4,0.8$ and 0.16 $\mu \mathrm{g} / \mathrm{mL}$ and incubated for 5 days at $37^{\circ} \mathrm{C}$, relative humidity $95 \%$ and $5 \% \mathrm{CO}_{2}$.

The intensity of colour resulting from the cleavage of CPRG by $T$. cruzi (Tulahuen C4) $\beta$-galactosidase was measured at $570 \mathrm{~nm}$ using a reader boards VersaMax Micro ${ }^{\mathrm{TM}}$ microplate reader. The $\mathrm{IC}_{50}$ of the compound were calculated by logarithmic regression of the values of OD obtained, compared with the untreated control. Those samples showing $\mathrm{IC}_{50}$ values $<50 \mu \mathrm{g} / \mathrm{mL}$, have been further tested for cytotoxicity. Nifurtimox (Bayer) was used as a control at concentrations of $0.1,1$ and 10 $\mu \mathrm{g} / \mathrm{mL}$. Negative Control was comprised of $50 \mathrm{uL}$ of a solution containing DMSO, equivalent to the DMSO contained in samples (working dilution).

\subsection{Biological Evaluation of the Synthesized Compounds}

All the synthesized 2-(substituted benzylidene)-5,7-dibromo-6-hydroxy-1-benzofuran-3(2H)-ones have been biologically evaluated against $T$. cruzi wherein two compounds $2 \mathbf{b}$ and $2 \mathbf{k}$ showed highest percentage Growth Inhibition (GI) 54.37 and 68.03 respectively. The $\mathrm{IC}_{50}$ value appeared as $8.37(\mu \mathrm{g} / \mathrm{mL})$ and $5.06(\mu \mathrm{g} / \mathrm{mL})$ for compounds $\mathbf{2 b}$ and $\mathbf{2 k}$ respectively with the standard drugs Nifurtimox and Benznidazole which were used to validate the assay. Cytotoxicity of compounds $2 \mathbf{b}$ (25 $\mu \mathrm{g} / \mathrm{mL})$ and $2 \mathbf{k}(37 \mu \mathrm{g} / \mathrm{mL})$ were also tested. The results are shown in Tables $\mathbf{1}$ and $\mathbf{2}$.

\section{Experimental}

\subsection{General}

All melting points (m.ps.) were determined in open capillaries on Veego (VMP-PM) melting point apparatus and are uncorrected. The purity of the compounds was routinely checked by thin layer chomatography (TLC) with Silica Gel-G (Merck). The instruments used for spectroscopic data are: IR-FTIR spectrophotometer Bruker, ${ }^{1} \mathrm{H}$ NMR and ${ }^{13} \mathrm{C}$ NMR $\left(\mathrm{CDCl}_{3}\right)$ on $500 \mathrm{MHz}$ FT-NMR spectrometer Bruker AV III, GC-MS (EI-MS fragment) performed on JEOL GC Mass spectrometer and elemental analysis was carried out on a Carlo Erba 1108 analyzer and were within the $\pm 0.5 \%$ of the theoretical values.

\subsection{General Procedure for the Synthesis of 2-(Substituted benzylidene)-5,7-dibromo- 6-hydroxyl-1-benzofuran-3(2H)-ones (2a-n)}

$\mathrm{CuBr}_{2}(10-15 \mathrm{mg})$ was dissolved in DMF-water mixture 


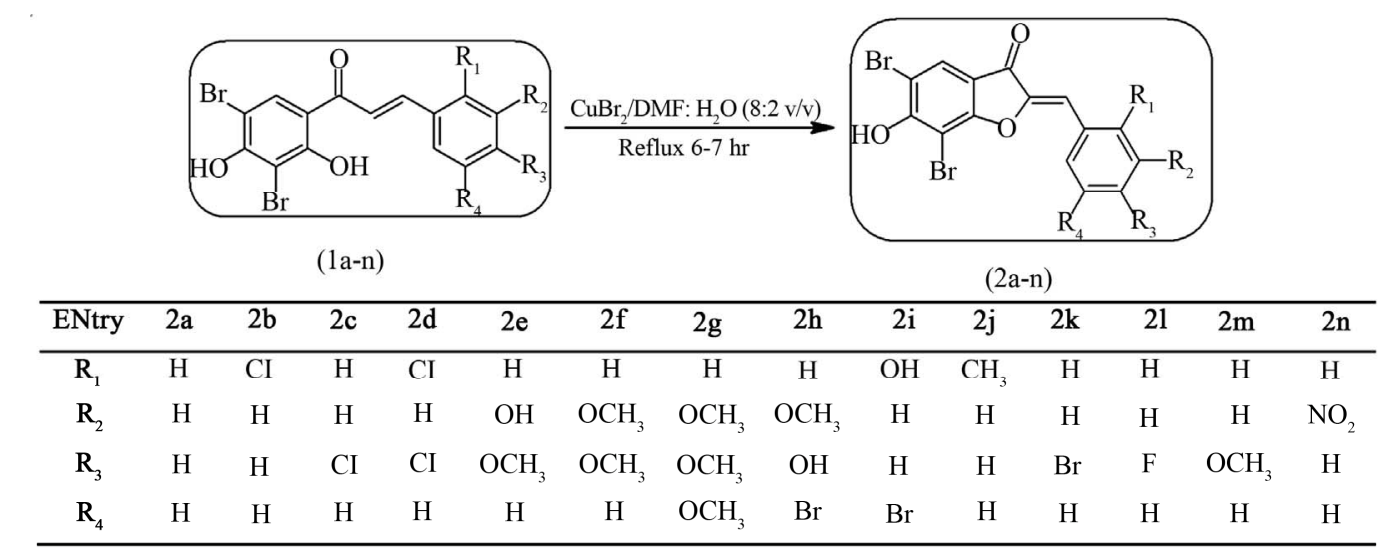

Scheme 1. Synthesis of the title compounds (2a-n). A novel series of some 2-(substituted benzylidene)-5,7-dibromo-6-hydroxy-1-benzofuran-3(2H)-ones 2 a-n was been synthesized by the oxidative cyclization of variously substituted chalcones using copper bromide in the presence of DMF-water mixture (8:2, v/v).

Table 1. Biological evaluation of 2-(substituted benzylidene)-5,7-dibromo-6-hydroxyl-1-benzofuran-3(2H)-ones against Trypanosoma cruzi; \% Growth inhibition.

\begin{tabular}{|c|c|c|c|c|c|}
\hline Entry & Concentration used & \% Growth Inhibition & Entry & Concentration Used & \% Growth Inhibition \\
\hline $2 a$ & $10 \mathrm{ug} / \mathrm{mL}$ & 15.72 & $2 \mathbf{i}$ & $10 \mathrm{ug} / \mathrm{mL}$ & 3.25 \\
\hline $2 b$ & $10 \mathrm{ug} / \mathrm{mL}$ & 54.37 & $2 \mathbf{j}$ & $10 \mathrm{ug} / \mathrm{mL}$ & 24.71 \\
\hline $2 c$ & $10 \mathrm{ug} / \mathrm{mL}$ & 20.18 & $2 \mathbf{k}$ & $10 \mathrm{ug} / \mathrm{mL}$ & 68.03 \\
\hline 2d & $10 \mathrm{ug} / \mathrm{mL}$ & 23.74 & 21 & $10 \mathrm{ug} / \mathrm{mL}$ & 19.63 \\
\hline $2 e$ & $10 \mathrm{ug} / \mathrm{mL}$ & 27.70 & $2 m$ & $10 \mathrm{ug} / \mathrm{mL}$ & 7.77 \\
\hline $2 f$ & $10 \mathrm{ug} / \mathrm{mL}$ & 16.15 & $2 n$ & $10 \mathrm{ug} / \mathrm{mL}$ & 3.86 \\
\hline $2 g$ & $10 \mathrm{ug} / \mathrm{mL}$ & 32.99 & Nifurtimox & $10 \mathrm{ug} / \mathrm{mL}$ & 68.50 \\
\hline $2 \mathbf{h}$ & $10 \mathrm{ug} / \mathrm{mL}$ & 4.12 & Benznidazole & $10 \mathrm{ug} / \mathrm{mL}$ & 86.77 \\
\hline
\end{tabular}

*Each value is the mean of three experiments.

Table 2. Biological evaluation of active samples against Trypanosoma cruzi; $\mathrm{IC}_{50}$ and Cytotoxicity.

\begin{tabular}{|c|c|c|c|c|}
\hline Entry & Concentration used & \% Growth Inhibition & ${ }^{* \mathrm{a}} \mathbf{I C}_{50}(\mathrm{ug} / \mathrm{mL})$ & ${ }^{\mathrm{b}}$ Cytotoxicity (ug/mL) \\
\hline $2 \mathbf{b}$ & $10 \mathrm{ug} / \mathrm{mL}$ & 54.37 & 8.37 & 25 \\
\hline $2 \mathbf{k}$ & $10 \mathrm{ug} / \mathrm{mL}$ & 68.03 & 5.06 & 37 \\
\hline Nifurtimox & $10 \mathrm{ug} / \mathrm{mL}$ & 68.50 & 0.47 & 27 \\
\hline Benznidazole & $10 \mathrm{ug} / \mathrm{mL}$ & 86.77 & 0.81 & $>50$ \\
\hline
\end{tabular}

${ }^{* \mathrm{a}} \mathrm{IC}_{50}$ : concentration that produces $50 \%$ inhibitory effect, ${ }^{\mathrm{b}}$ cytotoxicity: dose required to produce of $50 \%$ cell LLCMK2.

$(8: 2, \mathrm{v} / \mathrm{v})$ and to this substituted 3',5'-dibromo-2',4'dihydroxychalcones 1a-n $(0.02 \mathrm{~mol})$ were added. The resulted solution was refluxed for $6-7 \mathrm{hr}$. After the completion of reaction (monitored by TLC) the reaction mixture was cooled, and diluted with $50 \%$ ice cold water. The product obtained was filtered and purified by recrystallization from ethanol afforded pure samples of 2a-n. We tried different DMF: water mixture ratio (5:5 $\mathrm{v} / \mathrm{v}, 6: 4 \mathrm{v} / \mathrm{v}, 7: 3 \mathrm{v} / \mathrm{v}, 8: 2 \mathrm{v} / \mathrm{v}$ ) and finally the ratio $8: 2 \mathrm{v} / \mathrm{v}$ was found most suitable with improved yield $(63 \%$ $73 \%$ ).

Characterizations of the synthesized products are as follows:

(2Z)-2-benzylidene-5,7-dibromo-6-hydroxy-1-benzo furan-3(2H)-one (2a): Light brown solid; yield, 63\%; mp $90^{\circ} \mathrm{C}-91^{\circ} \mathrm{C}$; IR spectrum, $v, \mathrm{~cm}^{-1}: 3377$ (Ar-OH), 3071, 3009 (Ar-H), $1699(-\mathrm{C}=\mathrm{O}), 1642(-\mathrm{C}=\mathrm{CH}), 862$ (C-Br), 754 (-C-O-C). ${ }^{1} \mathrm{H}$ NMR spectrum $(500 \mathrm{MHz}$, 
$\left.\mathrm{CDCl}_{3}\right), \delta$, ppm, $(J, \mathrm{~Hz}): 9.93$ (s, 1H, Ar-OH), 7.95 (s, 1H, Ar-H), 7.42 - 7.08 (m, 5H, Ar-H), 6.77 (s, 1H, =CHPh). ${ }^{13} \mathrm{C}$ NMR $\left(500 \mathrm{MHz}, \mathrm{CDCl}_{3}\right)$ ppm: 190.27, 157.91, $152.93,147.99,137.43,135.21,130.28,129.82,126.05$, 114.62, 110.15, 105.62, 78.22. MS, $m / z: 395.80\left(\mathrm{M}^{+}\right)$. Anal. Calcd For $\mathrm{C}_{15} \mathrm{H}_{8} \mathrm{Br}_{2} \mathrm{O}_{3}$ : Found, \%: C, 45.52; H, 2.10. Calculated, \%: C, 45.48; H, 2.02.

(2Z)-5,7-dibromo-2-(2-chlorobenzylidene)-6-hydrox y-1-benzofuran-3(2H)-one (2b): Light brown solid; yield, $65 \%$; mp $193^{\circ} \mathrm{C}-194^{\circ} \mathrm{C}$; IR spectrum, $v, \mathrm{~cm}^{-1}$ : 3374 (Ar-OH), 3084, 3005 (Ar-H), 1701 (-C=O), 1641 $(-\mathrm{C}=\mathrm{CH}), 862(\mathrm{C}-\mathrm{Br}), 753(\mathrm{C}-\mathrm{Cl}), 758$ (-C-O-C). ${ }^{1} \mathrm{H}$ NMR spectrum $\left(500 \mathrm{MHz}, \mathrm{CDCl}_{3}\right), \delta, \mathrm{ppm},(J, \mathrm{~Hz}): 9.89$ (s, 1H, Ar-OH), 7.89 (s, 1H, Ar-H), 7.48 - 7.17 (m, 4H, Ar-H), 6.77 (s, 1H, =CHPh). ${ }^{13} \mathrm{C}$ NMR $(500 \mathrm{MHz}$, $\mathrm{CDCl}_{3}$ ) ppm: 188.49, 159.26, 155.66, 139.16, 135.21, 130.28, 129.60, 125.37, 114.68, 103.62, 79.19, 76.15. MS, $m / z: 430.30\left(\mathrm{M}^{+}\right)$. Anal. Calcd For $\mathrm{C}_{15} \mathrm{H}_{7} \mathrm{Br}_{2} \mathrm{O}_{3} \mathrm{Cl}$ : Found, \%: C, 41.87; H, 1.70. Calculated, \%: C, 41.83; H, 1.62 .

(2Z)-5,7-dibromo-2-(4-chlorobenzylidene)-6-hydrox y-1-benzofuran-3(2H)-one (2c): Brown solid; yield, $64 \%$; mp $99^{\circ} \mathrm{C}-100^{\circ} \mathrm{C}$; IR spectrum, $v, \mathrm{~cm}^{-1}: 3364$ $(\mathrm{Ar}-\mathrm{OH}), 3083,3013(\mathrm{Ar}-\mathrm{H}), 1705 \quad(-\mathrm{C}=\mathrm{O}), 1646$ $(-\mathrm{C}=\mathrm{CH}), 862$ (C-Br), 763 (-C-O-C), $753(\mathrm{C}-\mathrm{Cl}) .{ }^{1} \mathrm{H}$ NMR spectrum $\left(500 \mathrm{MHz}, \mathrm{CDCl}_{3}\right), \delta$, ppm, $(J, \mathrm{~Hz}): 9.91$ (s, 1H, Ar-OH), 7.73 (s, 1H, Ar-H), 7.78 - 7.27 (m, 4H, Ar-H), 6.89 (s, 1H, =CHPh). ${ }^{13} \mathrm{C}$ NMR $(500 \mathrm{MHz}$, $\mathrm{CDCl}_{3}$ ) ppm: 186.49, 158.26, 156.61, 137.16, 134.21, 130.38, 123.37, 114.81, 105.62, 78.19, 76.77, 64.19. MS, $m / z$ : $430.30\left(\mathrm{M}^{+}\right)$. Anal. Calcd For $\mathrm{C}_{15} \mathrm{H}_{7} \mathrm{Br}_{2} \mathrm{O}_{3} \mathrm{Cl}$ : Found, $\%$ : C, 41.78; H, 1.67. Calculated, \%: C, 41.83; H, 1.62 .

(2Z)-5,7-dibromo-2-(2,4-dichlorobenzylidene)-6-hy droxy-1-benzofuran-3(2H)-one (2d): Brown yellow solid; yield, $69 \%$; mp $219^{\circ} \mathrm{C}-220^{\circ} \mathrm{C}$; IR spectrum, $v$, $\mathrm{cm}^{-1}$ : 3390 (Ar-OH), 3078, 3005 (Ar-H), 1699 (-C=O), $1648(-\mathrm{C}=\mathrm{CH}), 862(\mathrm{C}-\mathrm{Br}), 783,753(\mathrm{C}-\mathrm{Cl}), 778$ (-C-OC). ${ }^{1} \mathrm{H}$ NMR spectrum $\left(500 \mathrm{MHz}, \mathrm{CDCl}_{3}\right), \delta$, ppm, $(J$, Hz): 9.97 (s, 1H, Ar-OH), 7.91 (s, 1H, Ar-H), 7.78 - 7.07 $(\mathrm{m}, 3 \mathrm{H}, \mathrm{Ar}-\mathrm{H}), 6.83$ (s, 1H, =CHPh). ${ }^{13} \mathrm{C} \mathrm{NMR}(500$ $\mathrm{MHz}, \mathrm{CDCl}_{3}$ ) ppm: 188.49, 157.26, 155.61, 135.16, 130.08, 125.37, 112.81, 104.62, 81.19, 76.77. MS, $m / z$ : $464.80\left(\mathrm{M}^{+}\right)$. Anal. Calcd For $\mathrm{C}_{15} \mathrm{H}_{6} \mathrm{Br}_{2} \mathrm{O}_{3} \mathrm{Cl}_{2}$ : Found, \%: C, 38.80; H, 1.35. Calculated, \%: C, 38.73; H, 1.29.

(2Z)-5,7-dibromo-6-hydroxy-2-(3-hydroxy-4-metho xybenzylidene)-1-benzofuran-3(2H)-one (2e): Light brown solid; yield, $65 \%$; mp $200^{\circ} \mathrm{C}-201^{\circ} \mathrm{C}$; IR spectrum, v, $\mathrm{cm}^{-1}$ : 3364 (Ar-OH), 3089, 3009 (Ar-H), 1707 (-C=O), $1638(-\mathrm{C}=\mathrm{CH}), 1034\left(-\mathrm{OCH}_{3}\right), 862(\mathrm{C}-\mathrm{Br}), 767(-\mathrm{C}-\mathrm{O}-\mathrm{C})$. ${ }^{1} \mathrm{H}$ NMR spectrum $\left(500 \mathrm{MHz}, \mathrm{CDCl}_{3}\right), \delta$, ppm, $(J, \mathrm{~Hz})$ : 10.12 (s, 1H, Ar-OH), 7.88 - 7.27 (m, 4H, Ar-H), 7.61 (s, $1 \mathrm{H}, \mathrm{Ar}-\mathrm{H}), 6.85(\mathrm{~s}, 1 \mathrm{H},=\mathrm{CHPh}), 3.73\left(\mathrm{~s}, 3 \mathrm{H},-\mathrm{OCH}_{3}\right)$. ${ }^{13} \mathrm{C}$ NMR $\left(500 \mathrm{MHz}, \mathrm{CDCl}_{3}\right)$ ppm: 189.49, 155.26, $152.31,139.96,134.78,115.37,111.27,80.39$, 77.27,
56.19. MS, $m / z$ : $441.80 \quad\left(\mathrm{M}^{+}\right)$. Anal. Calcd. for $\mathrm{C}_{16} \mathrm{H}_{10} \mathrm{Br}_{2} \mathrm{O}_{5}$ : Found, \%: C, 43.93; H, 3.69. Calculated, \%: C, 43.83; H, 3.62 .

(2Z)-5,7-dibromo-2-(3,4-dimethoxybenzylidene)-6-h ydroxy-1-benzofuran-3(2H)-one (2f): Brown solid; yield, $70 \%$; mp $129^{\circ} \mathrm{C}-130^{\circ} \mathrm{C}$; IR spectrum, $v, \mathrm{~cm}^{-1}$ : 3389 (Ar-OH), 3069, 3012 (Ar-H), $1711(-\mathrm{C}=\mathrm{O}), 1648$ $(-\mathrm{C}=\mathrm{CH}), 862(\mathrm{C}-\mathrm{Br}), 754$ (-C-O-C). ${ }^{1} \mathrm{H}$ NMR spectrum $\left(500 \mathrm{MHz}, \mathrm{CDCl}_{3}\right), \delta$, ppm, $(J, \mathrm{~Hz}): 9.85$ (s, 1H, Ar-OH), 7.98 - 7.41 (m, 3H, Ar-H), 7.84 (s, 1H, Ar-H), 6.87 (s, $1 \mathrm{H},=\mathrm{CHPh}), 3.86\left(\mathrm{~s}, 6 \mathrm{H}, \mathrm{Ar}-\mathrm{OCH}_{3}\right) \cdot{ }^{13} \mathrm{C} \mathrm{NMR}(500$ $\left.\mathrm{MHz}, \mathrm{CDCl}_{3}\right)$ ppm: 189.21, 153.38, 149.31, 137.86, 134.70, 116.49, 107.74, 79.31, 76.76, 57.74. MS, $m / z$ : $455.80\left(\mathrm{M}^{+}\right)$. Anal. Calcd. for $\mathrm{C}_{17} \mathrm{H}_{12} \mathrm{Br}_{2} \mathrm{O}_{5}$ : Found, \%: $\mathrm{C}$, 44.87; H, 2.58. Calculated, \%: C, 44.76; H, 2.63.

(2Z)-5,7-dibromo-6-hydroxy-2-(3,4,5-trimethoxybe nzylidene)-1-benzofuran-3(2H)-one (2g): Light yellow solid; yield, $71 \%$; mp $149^{\circ} \mathrm{C}-150^{\circ} \mathrm{C}$; IR spectrum, $v$, $\mathrm{cm}^{-1}$ : 3399 (Ar-OH), 3059, $3005(\mathrm{Ar}-\mathrm{H}), 1706(-\mathrm{C}=\mathrm{O})$, $1649(-\mathrm{C}=\mathrm{CH}), 862$ (C-Br), $786(-\mathrm{C}-\mathrm{O}-\mathrm{C}) .{ }^{1} \mathrm{H}$ NMR spectrum $\left(500 \mathrm{MHz}, \mathrm{CDCl}_{3}\right), \delta, \mathrm{ppm},(J, \mathrm{~Hz}): 9.87(\mathrm{~s}, 1 \mathrm{H}$, Ar-OH), 7.98 - 7.35 (d, 2H, Ar-H), 7.89 (s, 1H, Ar-H), $6.83(\mathrm{~s}, 1 \mathrm{H},=\mathrm{CHPh}), 3.83\left(\mathrm{~s}, 9 \mathrm{H}, \mathrm{Ar}-\mathrm{OCH}_{3}\right) .{ }^{13} \mathrm{C} \mathrm{NMR}$ (500 MHz, $\mathrm{CDCl}_{3}$ ) ppm: 189.29, 152.39, 148.51, 138.86, 135.70, 117.41, 108.74, 79.31, 77.76, 55.44. MS, $m / z$ : $485.80\left(\mathrm{M}^{+}\right)$. Anal. Calcd. for $\mathrm{C}_{18} \mathrm{H}_{14} \mathrm{Br}_{2} \mathrm{O}_{6}$ : Found, \%: C, 44.55; H, 2.76. Calculated, \%: C, 44.46; H, 2.88 .

(2Z)-5,7-dibromo-2-(3-bromo-4-hydroxy-5-methoxy benzylidene)-6-hydroxy-1-benzofura-3(2H)-one (2h): Light brown solid; yield, $68 \%$; mp $169^{\circ} \mathrm{C}-170^{\circ} \mathrm{C}$; IR spectrum, v, cm ${ }^{-1}$ : $3392(\mathrm{Ar}-\mathrm{OH}), 3059,3006(\mathrm{Ar}-\mathrm{H})$, $2848\left(-\mathrm{OCH}_{3}\right), 1709(-\mathrm{C}=\mathrm{O}), 1643(-\mathrm{C}=\mathrm{CH}), 862(\mathrm{C}-\mathrm{Br})$, 783 (-C-O-C). ${ }^{1} \mathrm{H}$ NMR spectrum $\left(500 \mathrm{MHz}, \mathrm{CDCl}_{3}\right), \delta$, ppm, $(J, \mathrm{~Hz}): 9.93,9.86(2 \mathrm{~s}, 2 \mathrm{H}, 2 \times \mathrm{Ar}-\mathrm{OH}), 7.91(\mathrm{~s}, 1 \mathrm{H}$, Ar-H), 7.82 - 7.87 (d, 2H, Ar-H), 6.83 (s, 1H, =CHPh), $3.83\left(\mathrm{~s}, 3 \mathrm{H},-\mathrm{OCH}_{3}\right) .{ }^{13} \mathrm{C} \mathrm{NMR}\left(500 \mathrm{MHz}, \mathrm{CDCl}_{3}\right) \mathrm{ppm}$ : 189.29, 155.19, 148.53, 141.11, 128.90, 115.41, 107.74, 81.92, 77.01, 55.44. MS, $m / z$ : $520.70\left(\mathrm{M}^{+}\right)$. Anal. Calcd. for $\mathrm{C}_{16} \mathrm{H}_{9} \mathrm{Br}_{3} \mathrm{O}_{5}$ : Found, \%: C, 36.94; H, 1.79. Calculated, $\%$ : C, 36.87; H, 1.73 .

(2Z)-5,7-dibromo-2-(5-bromo-2-hydroxybenzylidene)6-hydroxy-1-benzofuran-3(2H)-one (2i): Brown solid; yield, $70 \%$; mp $182^{\circ} \mathrm{C}-183^{\circ} \mathrm{C}$; IR spectrum, $v, \mathrm{~cm}^{-1}$ : 3394 (Ar-OH), 3054, 3009 (Ar-H), 1701 (-C=O), 1641 $(-\mathrm{C}=\mathrm{CH}), 862(\mathrm{C}-\mathrm{Br}), 786(-\mathrm{C}-\mathrm{O}-\mathrm{C}) .{ }^{1} \mathrm{H}$ NMR spectrum $\left(500 \mathrm{MHz}, \mathrm{CDCl}_{3}\right), \delta, \mathrm{ppm},(J, \mathrm{~Hz}): 9.91,9.78(2 \mathrm{~s}, 2 \mathrm{H}$, Ar-OH), 7.93 (s, 1H, Ar-H), 7.89 - 7.91 (m, 3H, Ar-H), $6.82(\mathrm{~s}, 1 \mathrm{H},=\mathrm{CHPh}) .{ }^{13} \mathrm{C} \mathrm{NMR}\left(500 \mathrm{MHz}, \mathrm{CDCl}_{3}\right) \mathrm{ppm}$ : 192.29, 156.57, 149.53, 137.11, 130.32, 114.34, 107.74, 76.76. MS, $m / z$ : $490.70\left(\mathrm{M}^{+}\right)$. Anal. Calcd. for $\mathrm{C}_{15} \mathrm{H}_{7^{-}}$ $\mathrm{Br}_{3} \mathrm{O}_{4}$ : Found, \%: C, 36.74; H, 1.48. Calculated, \%: C, 36.68; H, 1.42 .

(2Z)-5,7-dibromo-6-hydroxy-2-(2-methylbenzyliden e)-1-benzofuran-3(2H)-one (2j): Brown solid; yield, 
$69 \%$; mp $139^{\circ} \mathrm{C}-140^{\circ} \mathrm{C}$; IR spectrum, $v, \mathrm{~cm}^{-1}: 3392$ (Ar-OH), 3061, 3007 (Ar-H), $1699 \quad(-\mathrm{C}=\mathrm{O}), 1644$ $(-\mathrm{C}=\mathrm{CH}), 862(\mathrm{C}-\mathrm{Br}), 783$ (-C-O-C). ${ }^{1} \mathrm{H}$ NMR spectrum $\left(500 \mathrm{MHz}, \mathrm{CDCl}_{3}\right), \delta$, ppm, $(J, \mathrm{~Hz}): 9.97(\mathrm{~s}, 1 \mathrm{H}, \mathrm{Ar}-\mathrm{OH})$, $7.96(\mathrm{~s}, 1 \mathrm{H}, \mathrm{Ar}-\mathrm{H}), 7.89-7.18(\mathrm{~m}, 4 \mathrm{H}, \mathrm{Ar}-\mathrm{H}), 6.87(\mathrm{~s}$, $1 \mathrm{H},=\mathrm{CHPh}), 2.68\left(\mathrm{~s}, 3 \mathrm{H},-\mathrm{CH}_{3}\right) \cdot{ }^{13} \mathrm{C}$ NMR $(500 \mathrm{MHz}$, $\mathrm{CDCl}_{3}$ ) ppm: $190.29,152.39,149.51,137.82,129.90$, 114.41, 106.74, 79.92, 76.77, 29.23. MS, $m / z: 409.80$ $\left(\mathrm{M}^{+}\right)$. Anal. Calcd. for $\mathrm{C}_{16} \mathrm{H}_{10} \mathrm{Br}_{2} \mathrm{O}_{3}$ : Found, \%: C, 46.90; $\mathrm{H}, 2.50$. Calculated, \%: C, 46.85; H, 2.44 .

(2Z)-5,7-dibromo-2-(4-bromobenzylidene)-6-hydro xy-1-benzofuran-3(2H)-one (2k): Light brown solid; yield, $68 \%$; $\mathrm{mp} 225^{\circ} \mathrm{C}-226^{\circ} \mathrm{C}$; IR spectrum, $\mathrm{v}, \mathrm{cm}^{-1}$ : 3406 (Ar-OH), 3061, 3007 (Ar-H), $1711(-\mathrm{C}=\mathrm{O}), 1648$ $(-\mathrm{C}=\mathrm{CH}), 862(\mathrm{C}-\mathrm{Br}), 781$ (-C-O-C). ${ }^{1} \mathrm{H}$ NMR spectrum $\left(500 \mathrm{MHz}, \mathrm{CDCl}_{3}\right), \delta$, ppm, $(J, \mathrm{~Hz}): 9.93$ (s, $\left.1 \mathrm{H}, \mathrm{Ar}-\mathrm{OH}\right)$, 7.96-7.31 (m, 4H, Ar-H), 7.93 (s, 1H, Ar-H), 6.89 (s, 1H, $=\mathrm{CHPh}) .{ }^{13} \mathrm{C}$ NMR $\left(500 \mathrm{MHz}, \mathrm{CDCl}_{3}\right)$ ppm: 191.19, 156.39, 149.51, 141.81, 129.90, 116.41, 108.74, 81.92, 78.72. MS, $m / z: 474.70 \quad\left(\mathrm{M}^{+}\right)$. Anal. Calcd For $\mathrm{C}_{15} \mathrm{H}_{7} \mathrm{Br}_{3} \mathrm{O}_{3}$ : Found, \%: C, 37.97; H, 1.54. Calculated, \%: C, 37.92; H, 1.47.

(2Z)-5,7-dibromo-2-(4fluorobenzylidene)-6-hydroxy -1-benzofuran-3(2H)-one (2l): Light brown solid; yield, $73 \%$; mp $180^{\circ} \mathrm{C}-181^{\circ} \mathrm{C}$; IR spectrum, $v, \mathrm{~cm}^{-1}: 3491$ (Ar-OH), 3083, 3013 (Ar-H), 1705 (-C=O), 1649 (-C= $\mathrm{CH}), 1244$ (C-F), 862 (C-Br), 763 (-C-O-C). ${ }^{1} \mathrm{H}$ NMR spectrum $\left(500 \mathrm{MHz}, \mathrm{CDCl}_{3}\right), \delta$, ppm, $(J, \mathrm{~Hz}): 9.83(\mathrm{~s}, 1 \mathrm{H}$, Ar-OH), 7.89 (s, 1H, Ar-H), 7.78 - 7.34 (m, 4H, Ar-H), $6.79(\mathrm{~s}, 1 \mathrm{H},=\mathrm{CHPh}) .{ }^{13} \mathrm{C}$ NMR $\left(500 \mathrm{MHz}, \mathrm{CDCl}_{3}\right) \mathrm{ppm}$ : 191.29, 154.26, 149.31, 137.86, 134.70, 116.49, 108.85, 79.31, 76.77, 56.14. MS, $m / z: 413.80\left(\mathrm{M}^{+}\right)$. Anal. Calcd For $\mathrm{C}_{15} \mathrm{H}_{7} \mathrm{Br}_{2} \mathrm{O}_{3} \mathrm{~F}$ : Found, \%: C, 43.57; H, 1.62. Calculated, \%: C, 43.50; H, 1.69 .

(2Z)-5,7-dibromo-6-hydroxy-2-(4-methoxybenzylid ene)-1-benzofuran-3(2H)-one (2m): Brown solid; yield, $69 \%$; mp $87^{\circ} \mathrm{C}-88^{\circ} \mathrm{C}$; IR spectrum, $v, \mathrm{~cm}^{-1}: 3402$ $(\mathrm{Ar}-\mathrm{OH}), 3059,3005 \quad(\mathrm{Ar}-\mathrm{H}), 1713 \quad(-\mathrm{C}=\mathrm{O}), 1649$ $(-\mathrm{C}=\mathrm{CH}), 859$ (C-Br), 781 (-C-O-C). ${ }^{1} \mathrm{H}$ NMR spectrum $\left(500 \mathrm{MHz}, \mathrm{CDCl}_{3}\right), \delta$, ppm, $(J, \mathrm{~Hz}): 9.90(\mathrm{~s}, 1 \mathrm{H}, \mathrm{Ar}-\mathrm{OH})$, $7.93(\mathrm{~s}, 1 \mathrm{H}, \mathrm{Ar}-\mathrm{H}), 7.84-7.28(\mathrm{~m}, 4 \mathrm{H}, \mathrm{Ar}-\mathrm{H}), 6.84(\mathrm{~s}$, $1 \mathrm{H},=\mathrm{CHPh}), 3.88\left(\mathrm{~s}, 3 \mathrm{H},-\mathrm{OCH}_{3}\right) .{ }^{13} \mathrm{C}$ NMR $(500 \mathrm{MHz}$, $\mathrm{CDCl}_{3}$ ) ppm: $189.27,157.93,148.02,137.47,128.59$, 115.19, 107.73, 79.03, 78.37, 55.17. MS, $m / z: 425.80$ $\left(\mathrm{M}^{+}\right)$. Anal. Calcd For $\mathrm{C}_{16} \mathrm{H}_{10} \mathrm{Br}_{2} \mathrm{O}_{4}$ : Found, \%: C, 45.15; $\mathrm{H}, 2.42$. Calculated, \%: C, 45.09; H, 2.35 .

(2Z)-5,7-dibromo-6-hydroxy-2-(3-nitrobenzylidene) -1-benzofuran-3(2H)-one (2n): Brown solid; yield, 71\%; $\mathrm{mp} 182^{\circ} \mathrm{C}-183^{\circ} \mathrm{C}$; IR spectrum, $v, \mathrm{~cm}^{-1}: 3390(\mathrm{Ar}-\mathrm{OH})$, 3061, 3006 (Ar-H), $1708(-\mathrm{C}=\mathrm{O}), 1639(-\mathrm{C}=\mathrm{CH}), 1531$ (Asy Ar- $\mathrm{NO}_{2}$ ), 1350 (Sym Ar-NO ${ }_{2}$ ), 862 (C-Br), 786 (-C-O-C). ${ }^{1} \mathrm{H}$ NMR spectrum ( $\left.500 \mathrm{MHz}, \mathrm{CDCl}_{3}\right), \delta$, ppm, $(J, \mathrm{~Hz}): 9.91(1 \mathrm{~s}, 1 \mathrm{H}, \mathrm{Ar}-\mathrm{OH}), 7.97(\mathrm{~s}, 1 \mathrm{H}, \mathrm{Ar}-\mathrm{H}), 7.87$ $7.34(\mathrm{~m}, 4 \mathrm{H}, \mathrm{Ar}-\mathrm{H}), 6.89(\mathrm{~s}, 1 \mathrm{H},=\mathrm{CHPh}) .{ }^{13} \mathrm{C}$ NMR $(500$
$\left.\mathrm{MHz}, \mathrm{CDCl}_{3}\right)$ ppm: 192.19, 163.27, 156.85, 147.25, 137.73, 130.34, 127.87, 122.28, 114.50, 108.95, 79.26, 77.29. MS, $m / z: 440.80 \quad\left(\mathrm{M}^{+}\right)$. Anal. Calcd For $\mathrm{C}_{15} \mathrm{H}_{7} \mathrm{Br}_{2} \mathrm{O}_{5} \mathrm{~N}$ : Found, \%: C, 40.90; H, 1.63; N, 3.13 . Calculated, \%: C, 40.83; H, 1.58; N, 3.17 .

\section{Conclusion}

Two noteworthy have emerged. Firstly, a novel series of 2-(substituted benzylidene)-5,7-dibromo-6-hydroxy-1-benzofuran-3(2H)-ones have been synthesized from corresponding chalcones using environmentally benign protocols. Secondly, it was observed from the results obtained by the trypanocidal evaluation that Compounds $\mathbf{2 b}$ and $2 \mathbf{k}$ were the more potent, showing good \% GI and considered as an active and selected for second level biological evaluation $\mathrm{IC}_{50}$. Compounds $\mathbf{2 b}$ and $2 \mathbf{k}$ were also tested for cytotoxicity and the results obtained are appreciable in accordance with standard drugs Nifurtimox and Benznidazole. These preliminary results of biological assay of the tested compounds are encouraging which may lead to the discovery of potent trypanocidal drug.

\section{Acknowledgements}

The authors are thankful to Dr. Sunil Jhakhoria, Dean, FASC, MITS University for his constant encouragement during this work. Authors are also thankful to the Head, Sophisticated Analytical Instrument Facility, Indian Institute of Technology, Madras for spectral analysis.

\section{REFERENCES}

[1] T. A. Geissmann, "The Chemistry of Flavonoids Compounds," Pergamon Press, Oxford, London, Vol. 100, No. 1, 1962, pp. 60-61.

[2] A. R. Katritzky, H. H. Odens, S. M. Zhang, C. J. Rostek and O. W. Maender, "Novel Syntheses of 2,3-Dihydro1,5-benzothiazepin-4(5H)-ones and 2H-1,4-benzothiazin3(4H)-ones," Journal of Organic Chemistry, Vol. 66, No. 20, 2001, pp. 6792-6796. doi:10.1021/jo0101959

[3] O. Kayser, A. F. Kiderlen and R. Brun, "In Vitro Activity of Aurones against Plasmodium Falciparum Strains K1 and NF54," Planta Medica, Vol. 67, No. 8, 2001, pp. 718-721. doi:10.1055/s-2001-18356

[4] O. Kayser, A. F. Kiderlen, U. Folkens and H. Kolodziej, "In Vitro Leishmanicidal Activity of Aurones," Planta Medica, Vol. 65, No. 4, 1999, pp. 316-319. doi:10.1055/s-1999-13993

[5] B. Malhotra, J. C. Onyillagha, B. A. Bohm, G. H. N. Towers, D. James, J. B. Harborne and C. J. French, "Inhibition of Tomato Ring-Spot Virus by Flavonoids," Phytochemistry, Vol. 43, No. 6, 1996, pp. 1271-1276. doi:10.1016/S0031-9422(95)00522-6

[6] N. Hadj-Esfandiari, L. Navidpour, H. Shadnia, M. Amini, N. Samadi, M. A. Faramarzi and A. Shafiee, "Synthesis, 
Antibacterial Activity, and Quantitative Structure-Activity Relationships of New (Z)-2(Nitroimidazolylmethlene)-3(2H)-bezofuranone Derivatives," Bioorganic and Medicinal Chemistry Letters, Vol. 17, No. 22, 2007, pp. 6354-6363. doi:10.1016/j.bmcl.2007.09.062

[7] N. Fujii, J. P. Mallari, E. J. Hansell, Z. Mackey, P. Doyle, Y. M. Zhou, J. Gut, P. J. Rosenthal, J. H. McKerrow and R. K. Guy, "Discovery of Potent Thiosemicarbazone Inhibitors of Rhodesain and Cruzain," Bioorganic and Medicinal Chemistry Letters, Vol. 15, No. 1, 2003, pp. 121-124. doi:10.1016/j.bmcl.2004.10.023

[8] R. Siles, S. E. Chen, M. Zhou, K. G. Pinney and M. L. Trawick, "Design, Synthesis, and Biochemical Evaluation of Novel Cruzain Inhibitors with Potential Application in the Treatment of Chagas' Disease," Bioorganic and Medicinal Chemistry Letters, Vol. 16, No. 16, 2006, pp. 4405-4409. doi:10.1016/j.bmcl.2006.05.041

[9] R. V. C. Guido, G. Oliva, C. A. Montanari and A. D. Andricopulo, "Structural Basis for Selective Inhibition of Trypanosomatid Glyceraldehyde-3-Phosphate Dehydrogenase: MolecuLar Docking and 3D QSAR Studies," Journal of Chemical Information and Modeling, Vol. 48, No. 4, 2008, pp. 918-929. doi:10.1021/ci700453j

[10] D. M. Borchhardt, A. Mascarello, L. D. Chiaradia, R. J. Nunes, G. Oliva, R. A. Yunes and A. D. Andricopulo, "Biochemical Evaluation of a Series of Synthetic Chalcone and Hydrazide Derivatives as Novel Inhibitors of Cruzain from Trypanosoma cruzi," Journal of Brzalian Chemical Society, Vol. 21, No. 1, 2010, pp. 142-150. doi:10.1590/S0103-50532010000100021

[11] P. M. Sivakumar, S. Ganesan, P. Veluchamy and M. Doble, "Novel Chalcones and 1,3,5-Triphenyl-2-pyrazoline Derivatives as Antibacterial Agents," Chemical Biology and Drug Design, Vol. 76, No. 5, 2010, pp. 407-411. doi:10.1111/j.1747-0285.2010.01020.x

[12] A. R. Trivedi, D. K. Dodiya, N. R. Ravat and V. H. Shah, "Synthesis and Biological Evaluation of Some New Pyrimidines via a Novel Chalcone Series," Arkivoc, Vol. xi, 2008, pp. 131-141.

[13] F. Herencia, M. L. Ferrandiz, A. Ubeda, J. N. Dominguez, J. E. Charris, G. M. Lobo and M. J. Alcaraz, "Synthesis and Anti-Inflammatory Activity of Chalcone Derivatives," Bioorganic and Medicinal Chemistry Letters, Vol. 8, No. 10, 1998, pp. 1169-1174. doi:10.1016/S0960-894X(98)00179-6

[14] X. Wu, P. Wilairat and M. L. Go, "Antimalarial Activity of Ferrocenyl Chalcones," Bioorganic and Medicinal Chemistry Letters, Vol. 12, No. 7, 2002, pp. 2299-2302. doi:10.1016/S0960-894X(02)00430-4

[15] J. H. Cheng, C. F. Hung, S. C. Yang, J. P. Wang, S. J. Won and C. H. Lin, "Synthesis and Cytotoxic, Anti-Inflammatory, and Anti-Oxidant Activities of 2',5'Dialkoxylchalcones as Cancer Chemopreventive Agents," Bioorganic and Medicinal Chemistry, Vol. 16, No. 15, 2008, pp. 7270-7278. doi:10.1016/j.bmc.2008.06.031

[16] Y. M. Lin, Y. Zhou, M. T. Flavin, L. M. Zhou, W. Nie and F. C. Chen, "Chalcones and Flavonoids as Anti-Tuberculosis Agents," Bioorganic and Medicinal Chemistry,
Vol. 10, No. 8, 2002, pp. 2795-2802. doi:10.1016/S0968-0896(02)00094-9

[17] S. S. Rao, U. S. Gahlot, S. S. Dulawat, R. Vyas, K. L. Ameta and B. L. Verma, "Microwave Induced Improved Synthesis and Anti-Bacterial Activity of Some Chalcones And Their 1-Acyl-3,5-diaryl-2-pyrazolines," Afinidad, Vol. 60, No. 505, 2003, pp. 271-276.

[18] S. Kothari, R. Vyas and B. L. Verma, "A Facile One Pot Conversion of 3',5'-Dibromo-4-Hydroxy Substituted Chalcone to Pyrimidine Derivatives and Their Antibacterial and Herbicidal Activity," Indian Journal of Heterocyclic Chemistry, Vol. 8, No. 4, 1999, pp. 285-288.

[19] U. S. Gahlot, S. S. Rao, S. S. Dulawat, K. L. Ameta and B. L. Verma, "A Facile One-Pot Microwave Assisted Conversion of 3'-5'-Dibromo/diiodo-4'-hydroxy Substitutes Chalcones to 2-Substituted-4,6-Diaryl Pyrimidines Using S-Benzylisothiouronium Chloride (SBT) and Their Antibacterial Activities," Afinidad, Vol. 60, No. 508, 2003, pp. 558-562.

[20] K. L. Ameta, Biresh Kumar, Nitu. S. Rathore and B. L. Verma, "Facile Synthesis of Some Novel 2-Substituted4,6-Diarylpyrimidines Using 4'-Hydroxy-3',5'-dinitro-chalcones and S-Benzylthiouronium Chloride," Organic Communication, Vol. 5, No. 1, 2012, pp. 1-11.

[21] A. Jiang, C. G.Yang, W. N. Xing and J. Wang, "Synthesis and Cytotoxicity of Evaluation of Novel Indolylpyrimidines and Indolylpyrazines as Potential Antitumor Agents," Bioorganic and Medicinal Chemistry, Vol. 9, 2001, pp. 1149-1154. doi:10.1016/S0968-0896(00)00337-0

[22] K. L. Ameta, B. Kumar and N. S. Rathore, "Microwave Induced Improved Synthesis of Some Novel Substituted 1, 3-Diarylpropenones and Their Antimicrobial Activity," E-Journal of Chemistry, Vol. 8, No. 2, 2011, pp. 665-671. doi: $10.1155 / 2011 / 165047$

[23] K. L. Ameta, N. S. Rathore and B. Kumar, "Synthesis of Some Novel Chalcones and Their Facile One-Pot Conversion to 2-Aminobenzene-1,3-dicarbonitriles Using Malononitrile," Analele Universitatii Bucuresti Chimie, Vol. 20, No. 1, 2011, pp. 15-24.

[24] K. L. Ameta, N. S. Rathore and B. Kumar, "Synthesis and in Vitro Anti Breast Cancer Activity of Some Novel 1,5Benzothiazepine Derivatives," Journal of Serbian Chemical Society, Vol. 77, No. 6, 2012, pp. 725-731. doi:10.2298/JSC110715219A

[25] G. Wagner and B. Eppner, "Synthesis of Amidinobenzylidene Derivatives of Coumaran-3-One, 5,6-Benzocoumaran-3-one and 1-Thiocoumaran-3-one," Pharmazie, Vol. 34, 1979, pp. 527-530.

[26] L. Farkas, M. Nógrádi and L. Pallos, "The Correct Structure and Synthesis of Rengasine," Tetrahedron Letters, Vol. 4, No. 28, 1963, pp. 1999-2000. doi:10.1016/S0040-4039(01)90957-8

[27] R. S. Varma and M. Varma, "Alumina-Mediated Condensation. A Simple Synthesis of Aurones," Tetrahedron Letters, Vol. 33, No. 40, 1992, pp. 5937-5940. doi:10.1016/S0040-4039(00)61093-6

[28] D. Villemin, B. Martin and N. Bar, "Application of Microwave in Organic Synthesis. Dry Synthesis of 2- 
Aryl-methylene-3(2)-naphthofuranones," Molecules, Vol. 3, No. 3, 1998, pp. 88-93. doi:10.3390/30300088

[29] N. N. Agrawal and P. A. Soni, "A New Process for the Synthesis of Aurones by Using Mercury (II) Acetate in Pyridine and Cupric Bromide in Dimethyl Sulfoxide," Indian Journal of Chemistry, Vol. 45B, No. 5, 2006, pp. 1301-1303.

[30] B. S. Dawane, S. G. Konda, N. T. Khandare, S. S. Chobe, B. M. Shaikh, R. G. Bodade and V. D. Joshi, "Synthesis and Antimicrobial Evaluation of 2-(2-Butyl-4-chloro-
$1 H$-imidazol-5-yl-methylene)-substituted-benzofuran-3ones," Organic Communication, Vol. 3, No. 2, 2010, pp. 22-29.

[31] F. S. Buckner, C. L. Verlinde, A. C. La Flamme and W. C. van Voorhis, "Efficient Technique for Screening Drugs for Activity against Trypanosoma cruzi Using Parasites Expressing $\beta$-Galactosidase," Antimicro Agents and Chemotherapy, Vol. 40, No. 11, 1996, pp. 2592-2597. 